\title{
Pushing the boundaries of computational approaches: special focus issue on computational chemistry and computer-aided drug discovery
}

\author{
"In light of the scientific diversity of the computational chemistry field and the \\ frequently observed heterogeneity of computational studies, initiatives to showcase \\ computational methods and applications with relevance for medicinal chemistry and \\ drug discovery are of critical importance for the further development of the field."
}

Keywords: computational chemistry • computer-aided drug discovery • medicinal chemistry - method development $\bullet$ prospective applications

This editorial introduces a new special focus issue dedicated to computational chemistry and computer-aided drug discovery to be published in the third quarter of 2016, for which the author will serve as a guest editor. To position this special issue some background information is provided and a few important aspects are highlighted.

Medicinal chemistry publications frequently contain computational studies. Many of these studies are descriptive in nature. For example, a computational model is added to an experimental structure-activity relationship (SAR) investigation. This typically leads to the formulation of hypotheses as to how a compound might interact with its target or why active compounds differ in their potency. Another popular exercise is virtual screening aiming to identify novel active compounds using structure- and/or ligand-based computational approaches. Less frequent are computational investigations reporting the prospective design of new compounds for synthesis and biological evaluation. In addition, compound property analyses are carried out, for example, to rationalize or predict drug-likeness or ADME characteristics. Furthermore, new computational concepts or methods are published to aid in drug discovery efforts at different levels.

Computational studies reported in the medicinal chemistry literature are often characterized by a high degree of scientific heterogeneity. For example, modeling of compound binding modes is a popular exercise, often carried out to rationalize SARs. However, such predictions are frequently over-interpreted and putative interactions are discussed at the atomic level of detail as if they were experimental observations. On the other hand, there are also careful studies that evaluate putative binding modes taking the accuracy limitations of modeling into account and formulate experimentally testable hypotheses. However, the situation becomes particularly delicate when 'multihypothetical' strategies are pursued. This is illustrated by considering other examples from structure-based modeling. It is not uncommon that homology models are built for targets of interest, active compounds are docked into modeled binding sites, binding (free) energies are calculated for these 'double-hypothetical' ligand-target complexes, and correlated with experimentally determined affinities. What can one conclude from such exercises if close correlation between computed binding energies and experimental data is ultimately reported? Do such findings 'validate' the computational approach? Or might they represent the 'luck of the draw'? Scientific views might well differ in such cases. Regardless, these types of investigations more or less follow a 'look what computational methods can do!' theme and often give incorrect impressions, especially to non-experts. This is far from being helpful for the further development of the field at

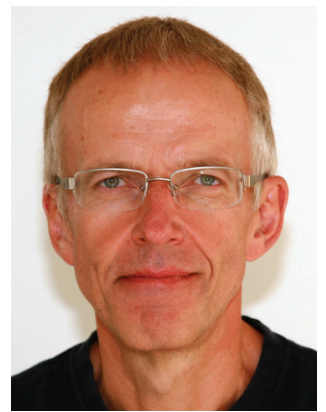

Jürgen Bajorath Department of Life Science Informatics, B-IT, LIMES Program Unit Chemical Biology \& Medicinal Chemistry, Rheinische Friedrich-

Wilhelms-Universität, Dahlmannstr. 2, D-53113 Bonn, Germany Tel.: +492282699306

Fax: +49 2282699341

bajorath@bit.uni-bonn.de 
interfaces with experimental disciplines. Problematic instances where science becomes science fiction are certainly not limited to computational approaches; however, they are particularly prone to this caveat when predictions do not take inherent accuracy limitations into account and disregard the realm of reality.

Pointing at controversial studies should by no means promote an overall negative view, as long as one is aware of these potential caveats. Rather, despite given accuracy limitations, there are many meaningful investigations and not all 'double-hypothetical' models are flawed per se. For example, homology models and docking calculations are also used to support iterative analog design by exploring alternative compound binding modes that can be probed - and often differentiated - through the introduction of specific functional groups in active compounds that might enhance or prevent binding. By testing such analogs, critical interactions can be identified and further explored and exploited to generate potent compounds. This leads directly to another important issue for computational approaches in drug discovery, in addition to scientific heterogeneity - the question of 'true' impact.

In medicinal chemistry, methods with clear-cut practical utility are particularly sought after. First and foremost, such methods should help to make better compounds. Demonstrating the ability of computational approaches to directly contribute to the identification and optimization of active compounds will typically spark the interest of medicinal chemists, more so than retrospective studies attempting to rationalize their findings. In this context, it should also be noted that the complexity of computational methods and the magnitude of calculations do not scale with the ability to impact experimental programs. Less is often more and the interpretability of computational findings plays a key role; if they can be translated into new experiments, an important hurdle is overcome. However, the issue of impact must also be viewed from different angles. Striving for practical utility cannot replace computational innovation, which requires the exploration of novel, often provocative, and off-thebeaten path concepts that might - at least initially - be far away from practical applicability. Despite the need for impact, computational chemists cannot be put into a purely supporting role in drug discovery settings, which prevents scientific progress to be made. Importantly, a meaningful balance must be found between practical utility and computational innovation, pushing the scientific boundaries of the field.

Computational chemistry is a highly diverse discipline. Below is a compendium of selected computational areas that are relevant for drug discovery research (in alphabetical order):
ADME/Tox analysis; chemical reaction modeling; de novo compound design; docking and scoring; dynamics of ligand-target interactions; free energy calculations; machine learning and compound classification; molecular property analysis; molecular similarity, dissimilarity and diversity analysis; pharmacophore methods; quantum mechanics/molecular mechanics; SAR and quantitative SAR (QSAR) analysis; structure-based design; virtual screening; visualization methods.

The list could be further refined and extended but already gives an impression of the scientific diversity of relevant computational areas.

In addition to these essentially established computational topics, there are other emerging areas that should also be considered. For example, the advent of 'big data' in drug discovery research $[1,2]$ is experiencing increasing interest. Without computational infrastructures and methods, it would not be possible to organize and archive exponentially growing amounts of compound activity and other chemical data and, importantly, extract knowledge from these data. Furthermore, computational approaches are being developed to systematically assess ligand-target interactions and explore polypharmacological networks or aid in target deconvolution from phenotypic screens.

In light of the scientific diversity of the computational chemistry field and the frequently observed heterogeneity of computational studies, initiatives to showcase computational methods and applications with relevance for medicinal chemistry and drug discovery are of critical importance for the further development of the field and for further increasing the impact of computational approaches. Journals such as Future Medicinal Chemistry [3-7] and the Journal of Medicinal Chemistry [8] should be commended on their efforts to highlight this important field with the launch of special computational issues. For our scientific community, these are milestone events that require our support as editors, authors, and interested readers.

The 2016 special focus issue on computational chemistry and computer-aided drug discovery continues the 'tradition' of computational focus issues in Future Medicinal Chemistry [3-7], with an expanded scope, given the computational topics discussed above. Manuscripts reporting computational methods and applications are encouraged that impact the entire spectrum of drug discovery efforts focusing on small molecules.

The deadline for submission to the special focus issue is the 1st of April, 2016. A range of articles will be considered including editorials, opinions, commentaries, preliminary communications, research articles, perspectives and reviews. We look forward to receiving many timely and interesting contributions from around the 
world, enabling Future Medicinal Chemistry to deliver an issue that highlights key challenges and demonstrates exciting progress that is being made in this field.

\section{Financial \& competing interests disclosure}

The author is an associated editor of the Journal of Medicinal Chemistry and a consultant to the pharmaceutical

\section{References}

$1 \mathrm{Hu} \mathrm{Y,} \mathrm{Bajorath} \mathrm{J.} \mathrm{Learning} \mathrm{from} \mathrm{'big} \mathrm{data':} \mathrm{compounds} \mathrm{and}$ Targets. Drug Discov. Today 19(4) 357-360 (2014).

2 Lusher SJ, McGuire R, van Schaik RC, Nicholson CD, de Vlieg J. Data-driven medicinal chemistry in the era of big data. Drug Discov. Today 19(7) 859-868 (2014).

3 Schneider G. Computational medicinal chemistry. Future Med. Chem. 3(4) 393-394 (2011).

4 D'Oca G. Computational medicinal chemistry: part II. Future Med. Chem. 3(6) 641-642 (2011). industry. The author has no other relevant affiliations or financial involvement with any organization or entity with a financial interest in or financial conflict with the subject matter or materials discussed in the manuscript apart from those disclosed.

No writing assistance was utilized in the production of this manuscript.

5 D'Oca G. Computational medicinal chemistry: part III. Future Med. Chem. 3(8) 899-900 (2011).

6 Future Medicinal Chemistry, volume 4, issue 15. www.future-science.com/toc/fmc/4/15

7 Schneider G. Breaking the data barrier in computational medicinal chemistry. Future Med. Chem. 6(3) 245-246 (2014).

8 Bajorath J, Jiang H, Shoichet BK, Walters WP. Computational methods for medicinal chemistry. J. Med. Chem. 58(3) 1019-1019 (2015). 